\title{
Atrapados en la marca-país
}

\author{
Rodrigo Berrios y Rodrigo Saens
}

RESUMEN

Sobre datos de 14.284 botellas provenientes de seis regiones/país: Argentina, Australia, Chile, California (Estados Unidos de América), Borgoña (Francia) y Sudáfrica, y cinco cosechas: 1997, 1999, 2001, 2004 y 2005, se estima un modelo de precios hedónicos que relaciona causalmente precio del vino con calidad individual y marca-país. Se confirma una relación positiva y estadísticamente significativa entre precio y calidad individual, y se detecta que el premio o castigo que reciben los vinos por marca-país —al igual que la elasticidad precio-calidadse ha mantenido estable en el tiempo. A igual calidad individual y respecto de los vinos californianos, los vinos chilenos y argentinos siguen siendo castigados hoy en más de un $50 \%$. Se constata también que el problema de la marca-país no tendrá solución mientras países entrantes a esta industria, como Chile o Argentina, no produzcan una masa crítica de vinos de excepcional calidad, factor que a fin de cuentas determina la consistencia de una buena imagen o reputación colectiva de sus productores.

PALABRAS CLAVE

CLASIFICACIÓN JEL

AUTORES
Vino, marcas comerciales, precios, calidad del producto, exportaciones, comercialización, datos estadísticos, Argentina, Chile, Australia, Francia, Sudáfrica, mercados, Estados Unidos

L15, D4, Q13

Rodrigo Berríos. Profesor Conferenciante Departamento de Administración, Universidad de Talca. roberrios@utalca.cl

Rodrigo Saens. Profesor Asistente Departamento de Economía y Finanzas, Universidad de Talca. rsaens@utalca.cl 


\section{I}

\section{Introducción}

La promisoria estrategia de entrada de las viñas chilenas en el mercado estadounidense en la década de 1990 - consistente en ofrecer un vino bueno y de bajo precio- ha dado paso 20 años después a una creciente frustración. En palabras de uno de los mejores viñateros de la industria: "los vinos chilenos son consumidos esencialmente por ser baratos".

Si el precio constituye una señal de calidad, es posible sugerir que la estrategia de entrada al mercado estadounidense, con una oferta inicial masiva de escasa diferenciación y bajo precio, conlleva el riesgo de prolongar de manera indefinida el período en que el vino chileno, al ser vinculado a la condición de vino barato, sea castigado por los consumidores con un precio menor al de su calidad sensorial u objetiva.

Es difícil que el consumidor discierna la calidad de un bien de experiencia como el vino, menos todavía del producido en un país poco conocido como Chile. Este más bien la infiere de su precio. El moderado éxito inicial de la estrategia chilena cuando no existían vinos baratos en el mercado estadounidense, así como su posterior estancamiento debido a la entrada de otros competidores con precios incluso más bajos — como los sudafricanos y los argentinos-, son dos fenómenos que pueden obedecer a la misma causa.

Diversas entidades han planteado la necesidad de acelerar la diferenciación del vino chileno sugiriendo tres vías para hacerlo: i) crear una imagen o marca-país que proporcione identidad y revalorice los diversos vinos producidos en Chile; ii) invertir más en innovación y calidad, como lo han hecho los australianos, y iii) cambiar las expectativas del consumidor estadounidense por medio de la producción de vinos de calidad mundial.

El objetivo de este trabajo es aportar evidencia empírica que permita evaluar el poder de marca de un país entrante como Chile. Utilizando el método de precios hedónicos de Rosen (1974), se analiza cuán sensible es el precio internacional del vino a indicadores de calidad y reputación. El estudio incluye cinco muestras en corte transversal de cinco cosechas entre los años 1997 y 2005, con indicadores publicados por Wine Spectator para vinos rojos producidos en la Argentina, Australia, los Estados Unidos de América (Napa y Sonoma), Chile, Francia (Borgoña) y Sudáfrica.
En términos de políticas públicas, el desafío de un país entrante es lograr que el consumidor evalúe sus distintos vinos en su propio mérito (calidad individual) y no la infiera de modo general de su débil imagen-país (reputación colectiva). Lograrlo no es fácil. De acuerdo con Roberts y Reagans (2007), al consumidor le es más cómodo evaluar los vinos más conocidos, como los franceses y los californianos, que los vinos de escasa presencia en la clasificación de calidad estadounidense, como los argentinos, chilenos o sudafricanos.

Los resultados de este trabajo revelan que no habrá solución para este problema mientras los países entrantes a esta industria, como Chile o la Argentina, no produzcan una masa crítica de vinos de excepcional calidad, que es a fin de cuentas lo que determina una buena imagen o reputación colectiva de sus productores.

$\mathrm{El}$ atajo de marketing de construir imagen sin calidad, no funciona. Incluso con una fuerte inversión en calidad, como en el caso de los australianos, construir reputación en el mercado del vino es un proceso lento y complejo, sobre todo si se trata de competir con los vinos franceses y californianos, que al construir poderosas y únicas asociaciones de imagen definieron y moldearon el significado cultural del vino moderno. Es precisamente esa inercia la que determina que los vinos chilenos y argentinos sigan sufriendo un fuerte castigo de precio.

Tampoco hay soluciones fáciles para esta inercia como las planteadas por Gibbs, Tapia y Warzynski (2009), quienes señalan que la globalización —con el inédito aumento de productos de procedencia no tradicional en el mercado estadounidense- ha acrecentado la proporción de consumidores que demandan más información acerca de la calidad objetiva del vino, incrementando la importancia de las clasificaciones de calidad individual publicadas por revistas especializadas como Wine Spectator. Si esta hipótesis fuera cierta, la elasticidad precio-calidad de los vinos aumentaría, y el castigo-país de los vinos chilenos o argentinos disminuiría con el tiempo. Los resultados aportados en este trabajo señalan evidencia en favor de lo contrario: la elasticidad precio-calidad parece estable para el período de estudio 1997-2005. El mercado sigue decidiendo por reputación.

Pero la pugna entre las principales marcas mundiales puede constituir una oportunidad para los países entrantes. Los resultados de este estudio muestran que Francia 
pierde poder de marca en comparación con California, y también en relación con algunos de los países entrantes. Una eventual causa de este fenómeno es un abuso de reputación de la marca dominante al final de su ciclo.

Este artículo se estructura de la siguiente forma. En la sección II se revisa brevemente la literatura. Los hechos estilizados, vinculados a indicadores de calidad y reputación de los vinos por país de origen, son explicados en la sección III. En la sección IV se presenta el modelo empírico y se analizan los principales resultados. Finalmente, en la sección V se resumen las principales conclusiones.

\section{II}

\section{¿lmagen o calidad?}

En el mercado del vino, una mayor calidad no es garantía de un mayor precio. La relación entre precio y calidad depende más de la percepción que tiene el consumidor respecto del país de origen del vino que de la calidad individual del producto. De acuerdo con los resultados de Brooks (2003) sobre la base de datos del mercado estadounidense, cualquier viñatero chileno o argentino obtiene solo la mitad del precio que logra un productor de vino de igual calidad, pero del valle de Napa, California.

Como señalan Costanigro, McCluskey y Mittelhammer (2007), la imagen de un país es una especie de bien público. La suerte de cualquier exportador vanguardista de Chile, que desee competir en el mercado premium de los Estados Unidos de América o de Europa, dependerá de la imagen que hayan proyectado los vinos chilenos en su conjunto, es decir, de aquello a lo que Tirole (1996) denomina reputación colectiva.

En este contexto, todos los vinos de un país entrante, buenos o regulares, son fuertemente castigados. La situación es peor aún para las pretensiones de los viñateros del Nuevo Mundo. Landon y Smith (1998) destacan cómo los precios de los vinos de Francia (Burdeos) en el mercado de los Estados Unidos de América se deben 20 veces más a su reputación pasada que a su calidad individual objetiva. La modificación de la imagen-país es lenta, y hay un marcado incentivo al abuso de reputación, práctica comercial que además pasará inadvertida por un buen tiempo.

Siguiendo a Tirole (1996) y Winfree y McCluskey (2005), la reputación vitivinícola la podemos entender como el prestigio, fama e imagen acumulados que se vinculan al nombre de un productor (reputación individual) o al de un conjunto de productores (reputación colectiva), como resultado de la trayectoria de calidad de sus vinos durante un considerable período de tiempo.

De acuerdo con Combris, Lecocq y M. Visser (1997) y Barber, Almanza y Donovan (2006), al ser el vino un bien de experiencia, el consumidor no es capaz de distinguir su calidad antes de probarlo. Es el prestigio asociado a una región o país el que le asegura al consumidor que los vinos de su procedencia sean de cierta calidad, marca-país que puede ser modificada solo en el margen y muy lentamente con la entrada de pocos vinos de calidad superior a la oferta ya existente.

Según Castriota y Delmastro (2008), el consumidor está dispuesto a pagar por dicho seguro un premio de precio, sobre todo si la región es de fama mundial, como Burdeos en Francia o Napa en California. Aún más, Lockshin y Rhodus (1993) y Schamel y Anderson (2003) señalan que la existencia de marcas agregadas de vino simplifica la vida del consumidor, que de otra forma se vería enfrentado a seleccionar una botella entre miles de opciones.

La misma dificultad que tiene el consumidor para distinguir calidad, le hará discriminar poco entre marcas individuales, facilitando su proliferación. Estas llegarán a ser miles en el mercado estadounidense, perdiendo su sentido como orientadoras de calidad del vino. En este escenario, a un productor no le es rentable invertir individualmente en crear poder de marca. Solo si deciden colaborar, los viñateros de una región estarán en mejores condiciones de afrontar estas inversiones y generar una marca colectiva poderosa, tal como - de acuerdo con Aylward y Zanko (2006) — lo hiciera California en los años setenta.

La mayoría de las otras regiones de procedencia, en particular la de los países entrantes, se convertirán en marcas agregadas por defecto, fundamentalmente porque generan una percepción de menor calidad vinculada a sus bajos precios, y en el mejor de los casos porque sugieren otro estilo de vino.

Brooks (2001 y 2003) lo ratifica al mostrar cómo en el mercado del vino los países funcionan como marcas colectivas, agregando un diferencial de precios que no 
puede ser explicado por ninguna otra variable. De este modo, un vino del valle de Napa en California logra, a igual calidad, el doble de precio que un vino chileno o argentino. Resultados similares son obtenidos por Schamel (2000 y 2002) y Schamel y Anderson (2003) para vinos del valle de Napa con respecto a los de Oregon, Washington, Nueva Zelandia, Australia, Chile, la Argentina y Sudáfrica ${ }^{1}$.

Como señalan Gergaud y Livat (2007) y Costanigro, McCluskey y Mittelhammer (2007), construir reputación, construir marca o ambos tienen como propósito alcanzar los mismos objetivos: diferenciar los vinos de una región para elevar su calidad percibida y lograr así un mejor precio.

\section{El caso de los países entrantes del fin del mundo}

Según Moguillansky, Salas y Cares (2006), la estrategia de value for money seguida por los grandes exportadores chilenos, consistente en ofrecer vinos de calidad media a precios menores que los ofrecidos por los productores europeos, independientemente de su éxito inicial —-logró captar el 5\% de las importaciones de este mercado en 20 años-, se tradujo en la concentración del $80 \%$ de los vinos chilenos en el segmento de bajos precios.

De acuerdo con Van Tienhoven (2008), esta imagen del vino chileno asociada a precios bajos es la única que ha podido percibir el grueso de los consumidores estadounidenses, estereotipándolo como un vino aceptable, pero solo para un uso corriente. Como explica Stein (2008), ello se ha convertido en una verdadera trampa de imagen para los productores, impidiendo que los consumidores paguen por la calidad efectiva del producto.

El consumidor estadounidense tiene poco tiempo e interés por averiguar la calidad individual de un vino. Para eso están las reputaciones. Según Schamel y Anderson (2003), los consumidores usan como regla heurística de compra la imagen estereotipada del país de origen del producto, más aún cuando compran vinos baratos.

El mercado refuerza continuamente estos estereotipos. El consumidor observa que en supermercados y tiendas especializadas los vinos argentinos, chilenos y sudafricanos se ubican en anaqueles donde se amontonan los vinos baratos; mientras que los californianos, y sobre todo los franceses, lo hacen en estantes en que se agrupan los vinos caros. Acostumbrado a vincular directamente calidad con precio, el consumidor aprende a relacionar la

\footnotetext{
${ }^{1}$ Que la reputación de Napa supere también a la de los estados de Oregon y Washington descarta que su premio se deba a variaciones en los costos de mano de obra con respecto a otros países, y muestra cómo las marcas por zona geográfica pueden circunscribirse solo a una región de un país.
}

calidad con el origen. Como señalan Lockshin y Rhodus (1993) y Chaney (2000): sea o no cierto, al país del que proviene el vino se le atribuye una calidad per se. En suma, un verdadero círculo vicioso para los entrantes.

\section{La muestra}

La muestra de 14.284 vinos rojos proviene de Wine Spectator. La baja presencia de vinos argentinos, chilenos y sudafricanos en WS refleja la realidad de su penetración en los Estados Unidos de América, que comienza recién en los años noventa. Es por esto que el análisis se inicia en la cosecha 1997, dado que antes no hubiera sido posible realizar estimaciones econométricas de los parámetros estudiados.

El método de muestreo utilizado es similar al de conglomerados, con una selección completa de todos sus elementos (vinos). Cada conglomerado es una región/ país elegida a priori por su papel en la dinámica de precios de este mercado. Burgundy (Borgoña), cuyos vinos tienen una importante y tradicional presencia en el mercado estadounidense, encarna la estrategia de exportación francesa. Los valles de Napa y Sonoma representan la exitosa estrategia ascendente de California, mientras que Australia, la Argentina, Chile y Sudáfrica la de aquellos países entrantes que en las últimas dos décadas han procurado mejorar su posicionamiento en los Estados Unidos de América.

Los disímiles tamaños de muestra resultante por cada región/país (véase el cuadro 1) reflejan el peso real que tienen sus vinos en el mercado estadounidense de vinos aceptables a excelentes - 70 a 100 puntos - cubierto por Wine Spectator. La variación del tamaño de muestra entre cosechas representa factores de oferta, demanda o de ambos, tales como los objetivos de volumen del Plan Australia.

El peso de los vinos de los países entrantes - también el de los franceses- en el universo de Wine Spectator es mayor que el que tienen en el mercado estadounidense real, donde de cada 10 vinos, 4 son de Napa y Sonoma, 2 del resto de California, 1 de otro estado estadounidense, y solo 3 son importados. Esto es así porque los países tienden a exportar sus mejores vinos.

Aunque la selección de solo algunas de las regiones/ país competidoras incide en que la muestra quede desproporcionada en favor de los vinos entrantes (4 de cada 10 vinos provienen de estos países), la inclusión de todos los vinos de cada región/país seleccionada hace que estas guarden entre sí su proporcionalidad original, permitiendo comparaciones realistas entre sus promedios de calidad y proporciones de vinos excelentes; a su vez, brinda tamaños de muestra robustos para las estimaciones econométricas. 
CUADRO 1

Muestra utilizada por país y año de cosecha

\begin{tabular}{lccccccc}
\hline \multirow{2}{*}{$\begin{array}{l}\text { Año } \\
\text { cosecha }\end{array}$} & \multicolumn{9}{c}{ País o región de origen } \\
\cline { 2 - 7 } & Argentina & Australia & California & Chile & Borgoña & Sudáfrica & Total cosecha \\
\hline 1997 & 76 & 358 & 1018 & 191 & 544 & 107 & 2294 \\
1999 & 114 & 514 & 1102 & 219 & 575 & 115 & 2639 \\
2001 & 120 & 624 & 1217 & 250 & 339 & 212 & 2762 \\
2004 & 328 & 674 & 1159 & 234 & 360 & 236 & 2991 \\
2005 & 358 & 622 & 1471 & 284 & 609 & 254 & 3598 \\
Total país & 996 & 2792 & 5967 & 1178 & 2427 & 924 & 14284 \\
\hline
\end{tabular}

Fuente: elaboración propia sobre la base de datos de Wine Spectator.

Nota: cada valor en el cuadro corresponde al número anual de vinos rojos por cada país/región utilizados en este estudio. El grueso del vino se vende a los dos y tres años después del año en que se cosecha.

\section{III}

\section{Los hechos estilizados: una interpretación}

En el cuadro 2, los indicadores de precio y calidad promedio muestran que la disposición a pagar de los consumidores, más que por la calidad objetiva del vino, está muy influenciada por la reputación, la imagen del país de origen o por ambas, señalando a los vinos franceses como aquellos que obtienen un mayor precio con

CUADRO 2

Evolución del precio y la calidad del vino proveniente
de seis países del mundo, 1997-2005

\begin{tabular}{|c|c|c|c|c|c|c|c|}
\hline \multirow{2}{*}{ Cosecha } & \multirow{2}{*}{ Indicador } & \multicolumn{6}{|c|}{ País o región de origen } \\
\hline & & Argentina & Australia & California & Chile & Borgoña & Sudáfrica \\
\hline \multirow[t]{3}{*}{1997} & Precio & 15,6 & 26,2 & 40,8 & 13,8 & 75,0 & 19,5 \\
\hline & Calidad & 81,4 & 86,4 & 87,6 & 82,9 & 83,5 & 84,5 \\
\hline & Razón & 0,20 & 0,30 & 0,47 & 0,17 & 0,90 & 0,23 \\
\hline \multirow[t]{3}{*}{1999} & Precio & 20,1 & 29,3 & 46,4 & 16,2 & 56,3 & 20,0 \\
\hline & Calidad & 84,5 & 86,5 & 87,6 & 83,8 & 84,7 & 84,6 \\
\hline & Razón & 0,24 & 0,34 & 0,53 & 0,19 & 0,66 & 0,24 \\
\hline \multirow[t]{3}{*}{2001} & Precio & 21,9 & 28,8 & 48,0 & 16,3 & 59,0 & 23,9 \\
\hline & Calidad & 84,1 & 86,8 & 86,0 & 83,4 & 86,3 & 85,4 \\
\hline & Razón & 0,26 & 0,33 & 0,56 & 0,19 & 0,68 & 0,28 \\
\hline \multirow[t]{3}{*}{2004} & Precio & 25,2 & 37,2 & 59,5 & 20,0 & 76,0 & 26,1 \\
\hline & Calidad & 85,6 & 88,0 & 87,2 & 84,5 & 87,9 & 85,8 \\
\hline & Razón & 0,30 & 0,42 & 0,68 & 0,24 & 0,86 & 0,30 \\
\hline \multirow[t]{3}{*}{2005} & Precio & 25,4 & 38,8 & 55,6 & 22,2 & 84,2 & 27,1 \\
\hline & Calidad & 85,3 & 88,6 & 86,9 & 85,6 & 89,9 & 85,6 \\
\hline & Razón & 0,30 & 0,44 & 0,64 & 0,26 & 0,99 & 0,32 \\
\hline
\end{tabular}

Fuente: elaboración propia sobre la base de datos de Wine Spectator.

Nota: los valores en el cuadro corresponden al promedio anual por país de cada indicador. El grueso del vino se vende a los dos y tres años después de la cosecha. La calificación de Wine Spectator fluctúa entre 50 puntos (muy malo) y 100 puntos (excelente). 
respecto a su calidad media, incluso si esta es menor que la de su principal retador, California, y similar a la de nuevos entrantes, como Chile, a fines de los años noventa.

Esto es más evidente en la evolución de la razón precio por unidad de calidad media de los vinos franceses, que sigue triplicando la de los vinos de países entrantes y representando una vez y media la de los californianos en 2005, al final del período analizado. En concordancia con Heslop, Cray y Armenakyan (2009), pareciera que nada hubiera cambiado en el imaginario del consumidor estadounidense, para el cual el vino francés sigue siendo sinónimo de excelencia.

Aunque al analizar más cuidadosamente la evidencia, resalta que algo comienza a cambiar con los vinos franceses a fines de los años noventa, cuando su razón precio/calidad desciende pronunciadamente de 0,9 a 0,68 entre 1997 y 2001, convergiendo hacia la razón ascendente del vino californiano, lo que sugería un debilitamiento tanto de su marca como de su premio de precio.

Pero esta baja fue solo temporal. A partir de 2001, los franceses reaccionan elevando sostenidamente su mediocre calidad promedio inicial, hasta alcanzar un extraordinario nivel de 90 puntos en 2005. Lograr esto les significó elevar sostenidamente la proporción de vinos excelentes de 90 puntos y más hasta un $44 \%$ —más allá que cualquier competidor-, y a la vez reducir drásticamente la exportación de vinos de débil calidad, tal como puede observarse en el cuadro 3 .
Este cambio no es producto del azar. Su explicación más plausible es la dificultad creciente de los franceses para seguir vendiendo vinos solo aceptables a dos y tres veces el precio de vinos equivalentes del Nuevo Mundo. En el análisis de Barco, Navarro y Langreo (2005) se apoya esta hipótesis, al afirmar que los vinos franceses vieron disminuir su participación en las importaciones estadounidenses de un $28 \%$ a un $14 \%$ entre 1993 y 2003, especialmente debido a la entrada de vinos australianos.

Cox y Bridwell (2007) redondean la hipótesis mostrando cómo, a partir de 1999, los franceses reposicionan exitosamente sus vinos en los segmentos de mayor calidad, elevando sus precios hasta el $100 \%$, al tiempo que abandonan los segmentos de menor calidad donde su estructura de costos no les permite competir.

Así, la razón final de precio/calidad media de 0,99 de los vinos franceses debe analizarse con cuidado, teniendo siempre en cuenta que esta se circunscribe a vinos muy buenos o excelentes, segmento donde los provenientes de Francia aún retienen su glamour. En la mayor parte del mercado, sin embargo, los vinos franceses pierden poder de marca frente a los californianos, así como su capacidad de imponer un premio de precio.

La paradoja inicial del vino francés, de baja calidad media y excelente reputación en 1997, es congruente con las teorizaciones de Tirole (1996). Un alto prestigio colectivo genera irresistibles incentivos para que algunos de sus productores, distribuidores o ambos vendan vinos de regular calidad a un elevado precio. Tal como ocurrió

Evolución de la cantidad de vinos de excelencia por país en el mercado californiano, 1997-2005

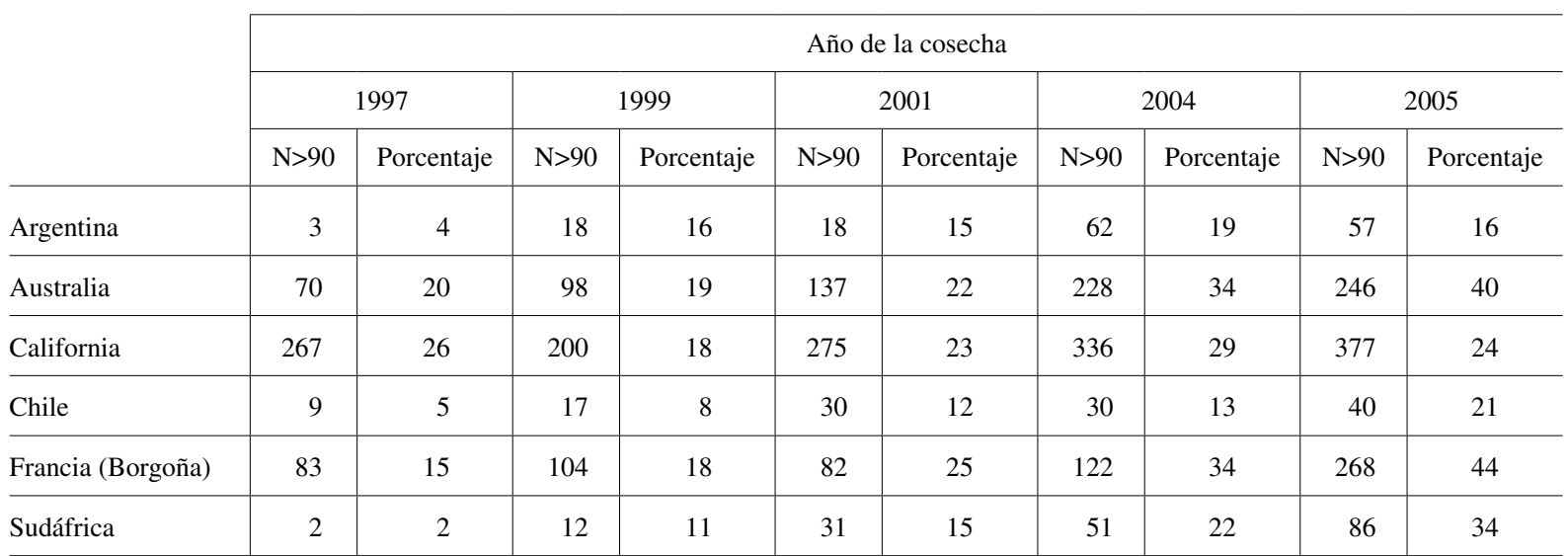

Fuente: elaboración propia sobre la base de datos de Wine Spectator.

Nota: los números en cada celda muestran la cantidad (N) y el porcentaje de vinos de cada país que obtienen 90 puntos o más en la calificación de Wine Spectator. 
a fines de los años noventa en los Estados Unidos de América, según reportan Landon y Smith (1997 y 1998).

De acuerdo con Roberts y Reagans (2007), es muy probable que el avance gradual en la transparencia del mercado del vino (mayor divulgación de las clasificaciones) haga menos tolerable para el consumidor seguir pagando más por malos vinos franceses en comparación con mejores vinos del Nuevo Mundo.

\section{El atajo del marketing}

Construir una reputación como la francesa, que dominó el mercado estadounidense por aproximadamente un siglo, es un camino prácticamente irreproducible. Como explican Hadj y Nauges (2007), dicha reputación fue moldeada desde 1855 mediante estrictas reglas de calidad que perduran hasta hoy, así como por medio de asociaciones de imagen que penetraron profundamente en el imaginario de los consumidores de ese país.

Desde el cine hollywoodense — con soldados saboreando un vino francés al fragor de la batalla por Europa - hasta el turismo masivo a sus castillos y viñedos, donde los estadounidenses adoptaron el lenguaje y la experiencia del vino, permitieron instalar asociaciones de imagen que proyectaron la fortaleza de marca francesa (Keller, 1993), logrando que el consumidor pagara por la experiencia de consumir sus vinos más allá de la calidad objetiva de éstos.

Es un mito que la ascendente construcción de marca de California haya tomado poco tiempo. Brosnan (2006) señala que a los mejores productores les llevó unos 30 años obtener la calidad y consistencia de los vinos franceses, pero casi un siglo adaptar lo mejor de su tradición a un contexto cultural único y glamoroso en el valle de Napa. Esto permitió que sus mejores vinos generaran beneficios emocionales más similares a los franceses, pero aparentemente solo para el público estadounidense.

La entrada de los vinos australianos en los años noventa les permitió adaptar la tecnología global igualando con rapidez la calidad de los vinos franceses y californianos. Su obstáculo fue la carencia de historia vitivinícola, que se intentó suplir con un marketing no convencional que vinculaba a los vinos australianos a conceptos como simpleza, amistad y honestidad, en contraposición al esnobismo del vino francés.

Tuvieron un éxito parcial. Según Heslop, Cray y Armenakyan (2009), el mérito de vinos entretenidos y baratos - como Yellow Tail_ fue atraer a consumidores cansados con la crítica especializada. Aunque el estereotipo fun de los vinos australianos generó volumen no produjo glamour ni precios acordes con su calidad, tampoco mejoró en nada su poder de marca frente a California.

En el cuadro 3 se indica la cantidad y proporción de vinos excepcionales — de más de 90 puntos en la calificación de Wine Spectator — producidos anualmente por los viñateros de cada región/país.

La comparación entre las proporciones corrobora el patrón ya esbozado de abuso de reputación, dominio excesivo por imagen o de ambos.

Mientras la proporción de vinos australianos excepcionales sube de un $20 \%$ en 1997 a un $40 \%$ en 2005, en California cae de un 26 a un 24\%. Los australianos muestran más compromiso con la calidad que los exitosos productores californianos, cuyo indicador es semejante al de países de bajo compromiso colectivo como la Argentina y Chile.

Estos resultados confirman las aprensiones de Shapiro (1983) sobre el estancamiento y erosión de la calidad media de una reputación colectiva exitosa. El valle de Napa, pese a producir la mayor cantidad de vinos excepcionales del mundo, atrajo el influjo masivo de productores beneficiarios parásitos (free riders) en busca de un premio de precio invocando solo su pertenencia al valle. Benjamin y Podolny (1999) estiman que un 50\% de las botellas etiquetadas como procedentes de Napa provienen en realidad de otros valles, amparados en la laxa legislación estadounidense de origen.

\section{La masa crítica}

La producción de una elevada cantidad absoluta de vinos de excelencia, al traducirse directamente en precios proporcionales a la reputación regional, apalanca la imagen de los países o regiones ícono.

California puede tener un bajo indicador de compromiso colectivo - y una menor calidad media frente a contendores como Australia-, pero su mayor cantidad absoluta de vinos excepcionales - 377 versus 246- además de su reputación ascendente, le aseguran el dominio del segmento de precios altos del mercado, emitiendo una importante señal de calidad para todos sus vinos. Como se ve en el cuadro 2, los precios extraordinarios los sigue dominando Francia con una menor, pero no despreciable, cantidad de vinos excepcionales.

Según Easingwood (2007), el consumidor utiliza la masa de vinos de precios elevados de una región/ país para inferir la calidad del resto de sus vinos. Esta masa crítica, además de interaccionar con la reputación, también la crea. Actualmente sirve más a California que a Francia, que ya no puede proyectar su influjo de marca a sus vinos de menor calidad. 
El cambio del poder de marca en la mayor parte del mercado estadounidense es sobre todo un cambio de imagen. California reemplaza a Francia, casi como Pepsi-Cola supera a Coca-Cola en las generaciones más jóvenes: es más cool. La calidad parece no importar tanto y el consumidor la entiende poco. De acuerdo con Costanigro, McCluskey y Goemans (2009), el mecanismo exacto por el cual una reputación emerge y se transforma en un mejor precio no es aún del todo conocido.
En suma, en términos de políticas públicas, los resultados del análisis descriptivo cuestionan la efectividad del marketing y la inversión en calidad como vías rápidas para librar a los productores de la trampa marca-país en la que se encuentran atascados. Tal como se desprende de los cuadros 2 y 3 , no hay atajos para zafar de este problema: la inercia en la imagen de marca creada por Francia, y más tarde por California, lo hace muy difícil.

\section{IV}

\section{Modelo empírico y resultados}

En la mayoría de los estudios empíricos que relacionan precio y calidad del vino se estiman variantes de la siguiente ecuación:

$$
\ln p_{i}=\beta_{0}+\beta_{1} \ln x_{i}+\beta_{2} \ln Y_{i}+\beta_{3} D_{j}
$$

donde $p_{i}$ es el precio de la botella $i, x_{i}$ es el puntaje de calidad individual (sensorial u "objetiva") que obtiene el vino después de una cata a ciegas, e $Y_{i}$ es un vector de variables de control, entre las que se encuentran la edad del vino, el monto producido y la reputación individual o marca del productor o viña. La elasticidad precio-calidad está dada por el coeficiente $\beta_{1}$, puesto que la especificación es doble logarítmica.

Landon y Smith (1997 y 1998) muestran cómo la omisión de variables de reputación regional o procedencia del vino sobreestima la importancia de la calidad en la determinación del precio, ya que su impacto en este es varias veces mayor.

Sobre la base del mismo enfoque, Schamel (2000), Schamel y Anderson (2003) y Costanigro, McCluskey y Goemans (2009) incorporan como predictores del precio del vino, además de la reputación del productor (reputación individual), la reputación del país o región de procedencia del vino (reputación colectiva).

Para calcular el premio o castigo (porcentual) en el precio de los vinos de un país con respecto al precio de los vinos de una región o país de referencia, se incorpora en la ecuación (1) una variable dicotómica, $D_{j}$, que toma el valor 1 si el vino es de la región/país "j” y 0 si no lo es. $\beta_{3}$ será positivo (negativo) y estadísticamente significativo cuando la reputación de la región/país, como productora de vino, es mayor (menor) que la de aquella utilizada como referente.
En el caso de este trabajo, la región/país utilizada como referente es California. Así, el valor absoluto de $\beta_{3}$ corresponde al premio o castigo (reputación colectiva), en porcentaje, del vino de la región/país respecto del vino californiano.

Modelos como el expresado en la ecuación (1) permiten medir, por separado, cuánto influyen la reputación del país de origen y la calidad efectiva del vino en la disposición a pagar del consumidor.

\section{Estimación del modelo}

Los datos para estimar la ecuación (1) provienen de cinco series en corte transversal informadas por Wine Spectator para las cosechas de 1997, 1999, 2001, 2004 y 2005. En estas series se incluyen: precio de mercado (en dólares corrientes de cada año), marca, número de cajas vendidas, edad, puntaje de calidad sensorial, país y región de origen de cada botella.

La edad se obtiene restando el año de su cosecha al año en que se evaluó el vino. Es de esperar que esta variable tenga un coeficiente con signo positivo. Dejar que un vino envejezca es una decisión de inversión: se opta por este camino cuando el incremento en el precio de venta, debido al aumento esperado en calidad, supera los costos (incluido el de capital) de almacenar el producto.

También es esperable que en la ecuación (1) la cantidad producida de vino, como variable explicativa en el vector $Y_{i}$, tenga un signo negativo: los consumidores intuyen que para producir grandes cantidades de un determinado vino, una viña deberá comprar uva en otros predios, perdiendo el control de la calidad del producto.

De acuerdo con Schamel (2000) y Costanigro, McCluskey y Mittelhammer (2007), la reputación del productor se mide como el número de vinos excepcionales 
-aquellos que obtienen un puntaje igual o superior a 90 puntos en la calificación de Wine Spectator - producidos por la viña en los últimos dos años.

\section{Los resultados}

En el cuadro 4 se presenta el resultado de cinco regresiones en corte transversal con que se analiza la relación entre precio, calificación de calidad, reputación individual y reputación colectiva por región/país en el mercado del vino. Dado que el precio y las demás variables no dicotómicas se encuentran todas expresadas en términos logarítmicos, los coeficientes pueden ser interpretados como elasticidades.

Las cinco regresiones muestran coeficientes para la elasticidad precio-calidad estadísticamente significativos, de signo positivo y de magnitud esperable, en el rango de los resultados reportados por Brooks (2001) y Schamel (2000).

Los resultados indican, además, que al contrario de lo presupuestado por Gibbs, Tapia y Warzynski (2009), la elasticidad precio-calidad se mantiene relativamente estable en el tiempo, oscilando entre 2,7 y 4,5 .

CUADRO 4

Evolución de la elasticidad precio-calidad y del premio (castigo) por país de origen en el mercado de vino de los Estados Unidos de América, 1997-2005

\begin{tabular}{|c|c|c|c|c|c|}
\hline \multirow{2}{*}{ Variables } & \multicolumn{5}{|c|}{ Año de la cosecha } \\
\hline & 1997 & 1999 & 2001 & 2004 & 2005 \\
\hline Constante & $\begin{array}{c}-12,257 \\
(11,8)\end{array}$ & $\begin{array}{l}-16,274 \\
(-17,5)\end{array}$ & $\begin{array}{c}-8,231 \\
(-10,5)\end{array}$ & $\begin{array}{l}-13,034 \\
(-14,5)\end{array}$ & $\begin{array}{l}-15,822 \\
(-17,4)\end{array}$ \\
\hline Elasticidad precio-calidad individual & $\begin{array}{c}3,545 \\
(15,3)\end{array}$ & $\begin{array}{c}4,533 \\
(21,9)\end{array}$ & $\begin{array}{c}2,735 \\
(15,6)\end{array}$ & $\begin{array}{c}3,826 \\
(19,1)\end{array}$ & $\begin{array}{c}4,504 \\
(22,2)\end{array}$ \\
\hline Elasticidad precio-reputación individual & $\begin{array}{l}0,125 \\
(9,2)\end{array}$ & $\begin{array}{l}0,081 \\
(6,8)\end{array}$ & $\begin{array}{l}0,150 \\
(13,6)\end{array}$ & $\begin{array}{c}0,126 \\
(11,9)\end{array}$ & $\begin{array}{c}0,121 \\
(12,2)\end{array}$ \\
\hline Marca Argentina & $\begin{array}{l}-0,331 \\
(-5,1)\end{array}$ & $\begin{array}{l}-0,433 \\
(-9,7)\end{array}$ & $\begin{array}{c}-0,475 \\
(-10,8)\end{array}$ & $\begin{array}{c}-0,559 \\
(-17,8)\end{array}$ & $\begin{array}{c}-0,546 \\
(-18,9)\end{array}$ \\
\hline Marca Australia & $\begin{array}{l}-0,193 \\
(-7,1)\end{array}$ & $\begin{array}{c}-0,322 \\
(-14,4)\end{array}$ & $\begin{array}{c}-0,406 \\
(-18,3)\end{array}$ & $\begin{array}{l}-0,426 \\
(17,2)\end{array}$ & $\begin{array}{c}-0,458 \\
(-19,1)\end{array}$ \\
\hline Marca Chile & $\begin{array}{l}-0,339 \\
(7,8)\end{array}$ & $\begin{array}{c}-0,382 \\
(-10,1)\end{array}$ & $\begin{array}{c}-0,599 \\
(-18,3)\end{array}$ & $\begin{array}{c}-0,597 \\
(-16,1)\end{array}$ & $\begin{array}{c}-0,564 \\
(-17,8)\end{array}$ \\
\hline Marca Francia & $\begin{array}{c}0,570 \\
(15,7)\end{array}$ & $\begin{array}{l}0,146 \\
(4,9)\end{array}$ & $\begin{array}{l}-0,092 \\
(-3,1)\end{array}$ & $\begin{array}{l}-0,135 \\
(-4,2)\end{array}$ & $\begin{array}{l}-0,225 \\
(-8,6)\end{array}$ \\
\hline Marca Sudáfrica & $\begin{array}{l}-0,435 \\
(-8,7)\end{array}$ & $\begin{array}{c}-0,496 \\
(-11,0)\end{array}$ & $\begin{array}{c}-0,496 \\
(-15,0)\end{array}$ & $\begin{array}{c}-0,540 \\
(-15,2)\end{array}$ & $\begin{array}{c}-0,612 \\
(-17,9)\end{array}$ \\
\hline Edad del vino & $\begin{array}{c}0,609 \\
(24,7)\end{array}$ & $\begin{array}{c}0,506 \\
(19,5)\end{array}$ & $\begin{array}{c}0,537 \\
(20,1)\end{array}$ & $\begin{array}{c}0,511 \\
(19,2)\end{array}$ & $\begin{array}{c}0,453 \\
(15,8)\end{array}$ \\
\hline Cajas producidas & $\begin{array}{c}-0,121 \\
(-17,2)\end{array}$ & $\begin{array}{c}-0,133 \\
(-22,5)\end{array}$ & $\begin{array}{c}-0,136 \\
(-24,8)\end{array}$ & $\begin{array}{c}-0,123 \\
(-21,3)\end{array}$ & $\begin{array}{c}-0,148 \\
(-27,9)\end{array}$ \\
\hline $\mathrm{R}^{2}$ ajustado & 0,64 & 0,63 & 0,63 & 0,62 & 0,58 \\
\hline Observaciones & 1842 & 2639 & 2646 & 2881 & 3407 \\
\hline
\end{tabular}

Fuente: elaboración propia sobre la base de datos de Wine Spectator.

Nota: los valores en las celdas muestran el parámetro estimado para cada variable en regresiones de corte transversal, una por cada año. La variable calidad corresponde al puntaje que obtiene el vino del productor en la calificación de Wine Spectator. El prestigio o reputación individual equivale al número de vinos con más de 90 puntos con la marca del productor en dicha clasificación. A excepción de las variables dicotómicas por país, todas las demás variables son medidas en logaritmo natural. El prestigio o marca-país se obtiene estimando el coeficiente de una variable dicotómica que toma el valor 1 si el vino es producido en el país y 0 si no lo es. Los números entre paréntesis corresponden al estadígrafo t-student. 
Asumiendo en el mejor de las casos que la elasticidad precio-calidad fuera 4,5 y que los viñateros de Chile aumentaran la calidad promedio en la calificación de Wine Spectator en un punto porcentual, acercándose a la calidad de los vinos del valle de Napa (tarea no fácil de realizar), el precio de mercado de los vinos chilenos subiría, en promedio, un 4,5\%, esto es, solo 1 dólar: de 22 a 23 dólares por botella. Así, elevar el precio promedio de los vinos de una región por la vía del mejoramiento de su calidad es un cambio demasiado lento.

Con respecto a la evolución de las marcas Francia y California, el consumidor estadounidense - que en la cosecha 1997 estaba dispuesto a pagar un 57\% más por vinos franceses de la misma calidad que los vinos californianos- disminuye rápidamente su disposición a pagar este premio de precio, lo que se observa con claridad en los coeficientes de la marca Francia: a partir de 2001, el premio francés desaparece y declina hasta alcanzar en 2005 una situación en que el consumidor pagará por estos vinos un $22 \%$ menos que por los vinos californianos de la misma calidad. Esto denota la decadencia de su poder de marca comparado con el de California.

No puede descartarse que Francia retenga poder de marca en el pequeño segmento de vinos extraordinarios, como sugieren los datos descriptivos, pero es complejo probarlo econométricamente debido a la escasez y poca varianza de calidad en este segmento, lo que excede los objetivos de este trabajo.

La Argentina, Chile y Sudáfrica no mejoran en nada su poder de marca, y sus vinos experimentan un castigo de precios relativamente similar. En particular, los consumidores castigan al vino chileno con un descuento creciente (en dólares por botella) que va de un $34 \%$ en 1997 a un $56 \%$ en 2005 , tal como lo muestran en el cuadro 4 sus respectivos coeficientes de marca-país.

La decadencia del poder de marca de los vinos chilenos está relativamente magnificada por el ascenso del poder de marca de California en desmedro de Francia. Si esto se corrige comparando los datos de Chile con los de Francia como indicador de referencia (benchmark), se constata que en realidad su castigo de precios en comparación con los vinos franceses de igual calidad incluso disminuye de un 96 a un $78 \%$ (esto se obtiene al sumar el valor absoluto de los coeficientes de ambos países).

Los resultados en el cuadro 4 reflejan también que el castigo de precios de Australia es solo un $10 \%$ menor que el de Chile en 2005. Este hallazgo no debe ser interpretado como muestra del desperdicio de esfuerzo de marketing y de calidad australiano; solo ilustra la complejidad de ganar poder de marca frente a las grandes reputaciones.

Considerando que Australia coloca tres veces más volumen y consigue, a igual calidad, un precio promedio un 10\% más alto que Chile, los vinos australianos tienen más poder de marca-país que sus competidores chilenos.

\section{La elasticidad precio-calidad por país}

La elasticidad precio-calidad obtenida es realmente un promedio entre las elasticidades precio-calidad de los distintos países analizados.

Con el objetivo de dilucidar si la evolución de este parámetro se comporta de manera similar o diferente entre países, se reestima el mismo modelo de la ecuación (1), pero esta vez con especificaciones separadas por país. La variable marca-país desaparece de la ecuación.

Si como señalan Gibbs, Tapia y Warzynski (2009), la globalización del mercado del vino incide en que la elasticidad precio-calidad aumente con el tiempo, la importancia de la marca-país disminuiría: los vinos deberían ser crecientemente evaluados por su calidad individual y menos por su región o país de origen.

Sin embargo, en el gráfico 1 se indica que esta elasticidad solo aumenta con claridad en el caso de Francia; para California, en cambio, la elasticidad precio-calidad baja en la medida en que la región gana reputación y poder de marca.

En el presente trabajo la explicación para este resultado tiene mucho que ver con el cambio de reputación y poder de marca entre ambas zonas de origen. El consumidor chequea escasamente la calidad del vino californiano: su creciente reputación le basta para pagar su precio. Por el contrario, los precios más elevados de los vinos franceses hacen que al consumidor le valga la pena incurrir en el costo de oportunidad de chequear sus precios.

El resto de los países analizados presenta una modesta tendencia al alza, una noticia favorable para los nuevos entrantes, pero absolutamente insuficiente en magnitud para inferir que el consumidor elige por calidad: este sigue eligiendo por marca-país.

\section{Explicando la imagen-país}

Las especificaciones analizadas en cuadro 4 y gráfico 1 monitorean la evolución del poder de marca de los distintos países competidores con un resultado negativo para los vinos del Nuevo Mundo: debido a una marca-país débil, la vía de mejorar la calidad individual para aumentar el precio promedio de sus vinos es muy lenta. 


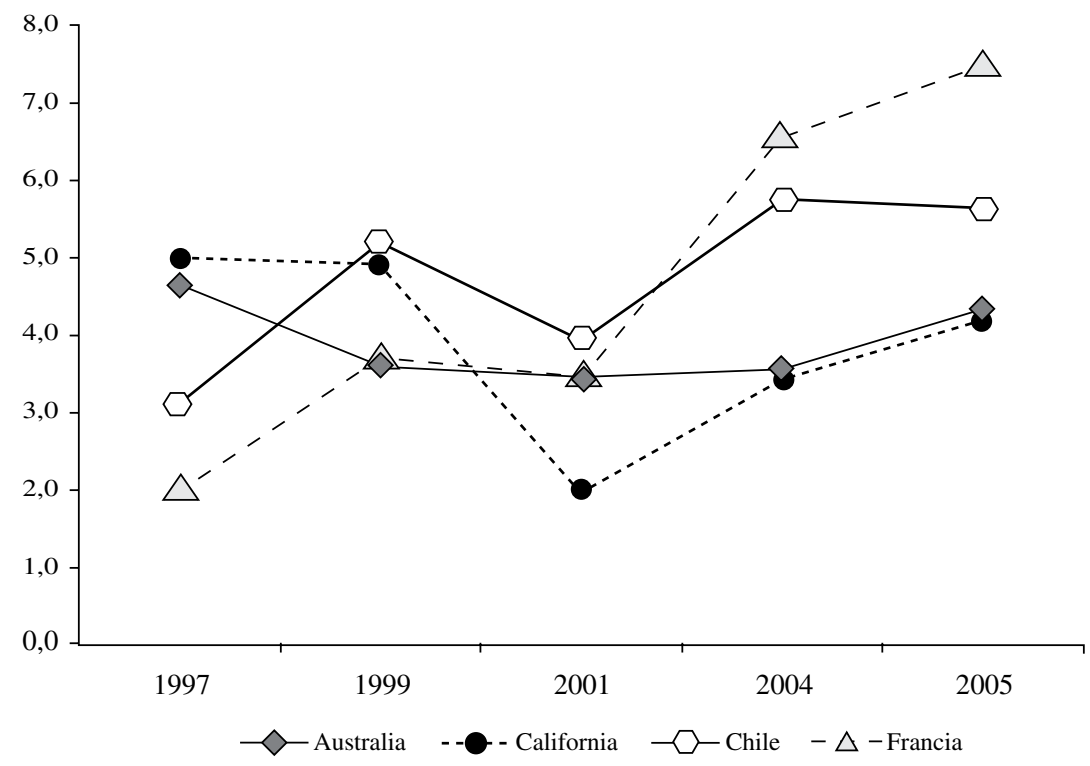

Fuente: elaboración propia sobre la base de datos de Wine Spectator.

El coeficiente que mide el poder de marca de una región no indica en qué medida este está determinado por la calidad efectiva (sensorial u objetiva) de sus vinos y en qué parte solo por su imagen. Ambos conceptos están mezclados y son difíciles de desentrañar.

En un intento por explicar qué significa el concepto de marca-país, se agrega una especificación adicional del modelo planteado en la ecuación (1): se sustituye la variable dicotómica que representa a cada región/ país por el número anual de vinos de excelencia ( 90 puntos o más) que logran los viñateros de un país en la calificación de Wine Spectator ${ }^{2}$.

El prestigio-país se convierte en variable. Es decir, varía entre los países según el número de vinos excelentes que produzca cada uno de estos y es constante para todos los vinos de un país. Así, como lo muestra el cuadro 3, una botella de vino para la muestra de 1997 tomará uno de los siguientes valores: 3, 70, 267, 9, 83 o 2, según

\footnotetext{
${ }^{2}$ La masa crítica o número de vinos de excelencia, además de ser intuitivamente razonable para explicar el prestigio o marca-país, es una variable econométricamente atractiva: constante para los vinos de un mismo país, pero altamente diferente entre países; covaría con el precio del vino y además muestra una baja correlación con el resto de las variables explicativas del modelo.
}

provenga de la Argentina, Australia, California, Chile, Francia, o Sudáfrica, respectivamente.

Evidentemente, en esta forma de replantear el modelo se asume que la reputación de un país que produce solo 9 vinos excelentes, como Chile en 1997, no puede ser sino muy baja en relación con una región como California que produce 267. Su objetivo es otro, de probarse la bondad de ajuste del modelo y la significancia estadística de esta nueva variable, sus resultados servirían para señalar la masa crítica de excelencia que un país entrante debiera tener a fin de derramar prestigio sobre el resto de sus vinos.

Los resultados reportados en el cuadro 5 muestran que la elasticidad precio-prestigio país, esto es, la sensibilidad del precio del vino al número de vinos excelentes producidos por los viñateros del país de origen del productor, además de ser estadísticamente significativa en todas las cosechas analizadas, sube aproximadamente de 0,1 en 1997 a 0,25 en 2005.

El precio del vino en el mercado estadounidense, fuertemente determinado por efectos de imagen previos a las cosechas del año 2000 — período del predominio francés-, pasará a tener un mayor componente de calidad: la masa crítica de excelencia. La imagen actuará a través de esta.

Si se considera que en la cosecha 2005 California tiene una masa crítica de vinos de excelencia 500\% 
CUADRO 5

Evolución de la elasticidad precio-calidad y de la elasticidad precio-marca-país en el mercado de vino de los Estados Unidos de América

\begin{tabular}{|c|c|c|c|c|c|}
\hline \multirow{2}{*}{ Variables } & \multicolumn{5}{|c|}{ Año de la cosecha } \\
\hline & 1997 & 1999 & 2001 & 2004 & 2005 \\
\hline Constante & $\begin{array}{l}-3,361 \\
(-3,34)\end{array}$ & $\begin{array}{l}-14,301 \\
(-15,41)\end{array}$ & $\begin{array}{l}-7,868 \\
(-9,76)\end{array}$ & $\begin{array}{r}12,361 \\
(-13,56)\end{array}$ & $\begin{array}{r}15,700 \\
(-16,91)\end{array}$ \\
\hline Elasticidad precio-calidad & $\begin{array}{r}1,575 \\
(6,87)\end{array}$ & $\begin{array}{r}3,943 \\
(18,83)\end{array}$ & $\begin{array}{r}2,394 \\
(13,28)\end{array}$ & $\begin{array}{r}3,369 \\
(16,38)\end{array}$ & $\begin{array}{r}4,085 \\
(19,68)\end{array}$ \\
\hline Elasticidad precio-marca productor & $\begin{array}{c}0,192 \\
(13,2)\end{array}$ & $\begin{array}{c}0,096 \\
(7,93)\end{array}$ & $\begin{array}{r}0,171 \\
(15,12)\end{array}$ & $\begin{array}{r}0,141 \\
(13,06)\end{array}$ & $\begin{array}{r}0,103 \\
(10,32)\end{array}$ \\
\hline Elasticidad precio-prestigio-país (número vinos $>90$ pts) & $\begin{array}{c}0,088 \\
(10,16)\end{array}$ & $\begin{array}{r}0,151 \\
(15,36)\end{array}$ & $\begin{array}{r}0,199 \\
(19,55)\end{array}$ & $\begin{array}{c}0,218 \\
(19,07)\end{array}$ & $\begin{array}{r}0,253 \\
(22,93)\end{array}$ \\
\hline Edad del vino & $\begin{array}{c}0,507 \\
(19,6)\end{array}$ & $\begin{array}{c}0,492 \\
(18,49)\end{array}$ & $\begin{array}{r}0,617 \\
(23,01)\end{array}$ & $\begin{array}{r}0,617 \\
(23,36)\end{array}$ & $\begin{array}{r}0,553 \\
(19,43)\end{array}$ \\
\hline Cajas producidas & $\begin{array}{c}-0,187 \\
(-28,3)\end{array}$ & $\begin{array}{r}-0,160 \\
(-28,96)\end{array}$ & $\begin{array}{r}-0,153 \\
(-28,84)\end{array}$ & $\begin{array}{r}-0,142 \\
(-25,73)\end{array}$ & $\begin{array}{r}-0,141 \\
(-27,22)\end{array}$ \\
\hline $\mathrm{R}^{2}$ ajustado & 0,57 & 0,59 & 0,60 & 0,59 & 0,62 \\
\hline Observaciones & 1842 & 2639 & 2646 & 2881 & 3407 \\
\hline
\end{tabular}

Fuente: elaboración propia sobre la base de datos de Wine Spectator.

Nota: los valores en las celdas muestran el parámetro estimado para cada variable en regresiones de corte transversal, una por cada año. La calidad individual corresponde al puntaje que logra el vino del productor en la calificación de Wine Spectator. Las variables prestigio individual y prestigio-país equivalen al número anual de vinos con más de 90 puntos logrados por el productor y los productores del país, respectivamente, en dicha calificación. Todas las variables son medidas en logaritmo natural. Los valores entre paréntesis corresponden al estadígrafo t-student.

mayor que la que tiene Chile — como se muestra en el cuadro 3 -, se puede concluir que los vinos californianos, en promedio, cuestan un $125 \%=0,253 \times 500 \%$ más que los vinos chilenos, resultado consistente con aquel mostrado en el cuadro 4: respecto a sus pares californianos, los vinos chilenos son castigados en poco más del $55 \%{ }^{3}$.

El incremento de la masa crítica de vinos de excelencia no solo acrecienta la calidad media de los vinos de un país, sino que también apalanca su imagen, lo

\footnotetext{
${ }^{3}$ Este porcentaje se obtiene al multiplicar 0,253 x 500\%.
}

que redunda en un aumento en el precio promedio de todos sus vinos. Si los viñateros de Chile doblaran la producción de vinos excepcionales obtenida en la cosecha 2005, pasando de 40 a 80 vinos, manteniéndose todo lo demás constante, el precio promedio de sus vinos se elevaría un $25 \%=0,253 \times 100 \%$, esto es, de 25 dólares a poco más de 31 dólares promedio cada botella.

Estos resultados muestran cómo un país que produce una gran cantidad de vinos excepcionales -mediante la exposición a la crítica profesional especializada- envía una poderosa señal de calidad al mercado, que resalta la percepción y precio de todos sus vinos. 


\section{V}

\section{Conclusiones y limitaciones}

Sobre la base de datos de vinos rojos del mercado estadounidense, el objetivo de este trabajo es evaluar, desde un punto de vista empírico, cuán importante es en el precio de mercado del vino la calidad individual del producto versus la reputación colectiva de su región o país de origen. Asimismo, se desea proporcionar elementos de políticas públicas que ayuden a países entrantes como la Argentina o Chile a fortalecer de manera más efectiva el posicionamiento de su marca-país.

Las estimaciones del modelo de precios hedónicos revelan que la marca-país ejerce todavía un efecto no menor en el precio de todos sus vinos, un tema difícil de resolver para un país entrante como Chile cuyo castigo de precios continúa casi al mismo nivel que a fines de los años noventa.

Si bien la mayor crítica a que se ven expuestos los productores ha redundado en que el mercado del vino sea hoy más transparente que en la década de 1990, ello solo ha erosionado parcialmente el papel de las reputaciones; de este modo, aunque redujo la de Francia, no sucedió lo mismo con la de California.

Al estimar el modelo por región/país, los resultados de este trabajo evidencian que, con excepción del caso de Francia, la elasticidad precio-calidad se muestra estable a través del tiempo y no creciente como sugieren Gibbs, Tapia y Warzynski (2009), reafirmando que el mercado sigue juzgando la calidad de los vinos más por su reputación colectiva que por su calidad individual, como lo sería en un mercado transparente.

El mensaje para los vinos de los países entrantes como Chile es inmediato: no es posible mejorar la marca país con el concepto "más calidad por un menor precio (value for money)". Pese a su éxito inicial de penetración, esta estrategia equivale a devaluar a priori la reputación de los buenos vinos chilenos.

Es extraordinariamente complejo lograr que el mercado valore los vinos entrantes. En efecto, los resultados de esta investigación permiten constatar que los productores de Francia antes, así como los de California hoy, ejercen su mayor poder de marca y consiguen mayores precios con una calidad media incluso inferior a la de los nuevos entrantes. Su poder ha consistido, y consiste, en una fuerte imagen colectiva.

En este trabajo también se detecta que si el mercado es opaco, la calidad media de la marca-país dominante tiene una tendencia natural a estancarse, o incluso a erosionarse, debido a la entrada de productores free riders que, aprovechándose de que proceden de una región reputada, venden vinos de baja calidad a un alto precio. Sin embargo, para países entrantes como la Argentina o Chile, esperar que una reputación colectiva dominante pueda erosionarse debido a esta causa es un camino lento e incierto.

Si bien, como señalan Roberts y Reagans (2007), las clasificaciones de vinos de revistas especializadas como Wine Spectator han ayudado a transparentar el mercado, la relación que mantiene el consumidor con estas publicaciones no es directa, está mediada por su precio y opera con rezago.

Esto explica que regiones como California puedan seguir dominando la mayor parte del mercado - imponiendo un premio de precio- al producir una gran masa de vinos de calidad excepcional, aunque su calidad regional promedio sea menor que la de otros contendores. La masa crítica absoluta de vinos excepcionales, más que la proporción de vinos excepcionales, es lo que da una señal de calidad o un halo de marca al consumidor respecto de todos los vinos de un país.

Los resultados obtenidos en este trabajo revelan que el aumento de la masa crítica de vinos excepcionales - de 90 puntos o más en la calificación de Wine Spectator - es un camino más rápido que el incremento de la calidad promedio para elevar la reputación, y con ello reforzar la relación precio-calidad promedio de los vinos de un país entrante. La reputación colectiva o marca de un país actúa como un bien público, y su premio o castigo se extiende a todos los vinos de la región/país.

Por lo tanto, más que utilizar el marketing para pretender - mediante la asociación de imágenes — cambiar la historia, sería más fructífero estimular y premiar (subsidiar) fuertemente la calidad excepcional de los vinos chilenos y argentinos hasta llegar a una masa crítica interesante. No habrá solución al problema de la marca-país mientras estos países entrantes no produzcan una masa crítica de vinos de excepcional calidad, factor que a fin de cuentas es el que determina la consistencia de una buena imagen o reputación colectiva de sus productores.

\section{Limitaciones del estudio}

Los datos de Wine Spectator presentan algunas debilidades. Al respecto, un escollo que presentan estas y otras 
investigaciones es que no hay absoluta claridad sobre como Wine Spectator determina los precios, y los volúmenes agregados por país no son tan precisos. Esto queda compensado, a nuestro juicio, por la posibilidad de hacer análisis causales respecto de la importancia de la marcapaís y la calidad individual en la determinación del precio.

\section{Bibliografía}

Aylward, D.K. y M. Zanko (2006), "Emerging Interorganizational Structures in the Australian Wine Industry: Implications for SMEs", Wollongong, Australia, Universidad de Wollongong.

Barber, N., B. Almanza y J. Donovan (2006), "Motivational factors of gender, income and age on selecting a bottle of wine", International Journal of Wine Marketing, vol. 18, No 3, Bingley, Reino Unido, Emerald.

Barco, E., M.C. Navarro y A. Langreo (2005), "Cambios en el mercado internacional del vino. Algunas preguntas sobre el éxito del vino australiano", Distribución y consumo, No 80, Madrid, Mercasa.

Benjamin, B.A y J.M. Podolny (1999), "Status, quality, and social order in the California wine industry", Administrative Science Quarterly, vol. 44, № 3, Ithaca, Cornell University.

Brooks, E. (2003), "Products and prejudice: measuring country of origin bias in U.S. wine imports", Santa Cruz Center for International Economics Working Papers, No 6228 , UC Santa Cruz.

(2001), "Countries as brands: international trade in wine", documento presentado en Enometrics VIII (Napa Valley, California, 21 a 22 de mayo), Giannini Foundation and Vineyard Data Qualification Society.

Brosnan, K. (2006), "Bidding on nature's quintessence: measuring the history of the Napa Valley wine auction", Working Paper, $\mathrm{N}^{\circ}$ 2006-005, París, European Association of Wine Economist.

Castriota, S. y M. Delmastro (2009), "The economics of collective reputation: minimum quality standards, vertical differentiation and optimal group size", AAWE Working Paper, $\mathrm{N}^{\circ}$ 50, Nueva York, American Association of Wine Economists.

(2008), "Individual and collective reputation: lessons from the wine market", Working Papers, No 45504, Nueva York, American Association of Wine Economists.

Chaney, I.M. (2000), "External search effort for wine", International Journal of Wine Marketing, vol. 12, $\mathrm{N}^{\mathrm{o}} 2$, Bingley, Reino Unido, Emerald.

Combris, P., S. Lecocq y M. Visser (1997), "Estimation of a hedonic price equation for Bordeaux wine: does quality matter?", The Economic Journal, vol. 107, № 441, Royal Economic Society.

Costanigro, M., J.J. McCluskey y C. Goemans (2009), “The economics of nested names: name specificity, reputation and price premia", AAWE Working Paper, $\mathrm{N}^{\circ}$ 49, Nueva York, American Association of Wine Economists.

Costanigro, M., J.J. McCluskey y R.C. Mittelhammer (2007), "Segmenting the wine market based on price: hedonic regression when different prices mean different products", Journal of Agricultural Economics, vol. 58, №3, Hoboken, Wiley Blackwell.

Cox, J. y L. Bridwell (2007), “Australian companies using globalization to disrupt the ancient wine industry", Competitiveness Review: An International Business Journal incorporating Journal of Global Competitiveness, vol. 17, № 4, Bingley, Reino Unido, Emerald.

Easingwood, Ch. (2007), "Positioning of wine regions: old or new world branding models?", documento presentado en el XIV Congreso de Econometría, Trier.

Gergaud, O. y F. Livat (2010), "Collective reputation effects: an empirical appraisal”, AAWE Working Paper, No 73, Nueva York, American Association of Wine Economists.

(2007), "How do consumers use signals to assess wine quality?", AAEW Working Paper, No 3, Nueva York, American Association of Wine Economists.
Gibbs, M., M. Tapia y F. Warzynski (2009), "Globalization, superstars, and reputation: theory and evidence from the wine industry", Journal of Wine Economics, vol. 4, № 1, Nueva York, American Association of Wine Economists.

Hadj, H. y C. Nauges (2007), "The pricing of experience goods: the example of En Primeur wine", American Journal of Agricultural Economics, vol. 89, № 1, Milwaukee, Agricultural \& Applied Economics Association.

Heslop, L., D. Cray y A. Armenakyan (2009), "Brand and country of origin effects in wine decision making: is incongruity a problem in a wine world turned upside down?", Proceedings of the Annual Conference of Administrative Sciences Association of Canada, Marketing Division (Niagara Falls, Ontario, 6 a 9 de junio).

Keller, K.L. (1993), "Conceptualizing, measuring, and managing customer-based brand equity", Journal of Marketing, vol. 57, $\mathrm{N}^{\mathrm{o}}$ 1, Chicago, American Marketing Association.

Landon, S. y C.E. Smith (1998), "Quality expectations reputation and price", Southern Economic Journal, vol. 64, № 3, Chattanooga, Tennessee, Southern Economic Association.

(1997), "The use of quality and reputation indicators by consumers: the case of Bordeaux wine", Journal of Consumer Policy, vol. 20, $\mathrm{N}^{\circ} 3$, Springer.

Lockshin, S.L. y W.T. Rhodus (1993), "The effect of price and oak flavor on perceived wine quality", International Journal of Wine Marketing, vol. 5, № 2-3, Bingley, Reino Unido, Emerald.

Moguillansky, G., J.C. Salas y G. Cares (2006), "Capacidad de innovación en industrias exportadoras de Chile", serie Comercio internacional, $N^{\circ} 79$ (LC/L.2619-P), Santiago de Chile, Comisión Económica para América Latina y el Caribe (CEPAL). Publicación de las Naciones Unidas, $\mathrm{N}^{\mathrm{o}}$ de venta: S.06.II.G.143.

Roberts, P. y R. Reagans (2007), "Critical exposure and price-quality relationships for new world wines in the U.S. market", Journal of Wine Economics, vol. 2, № 1, Nueva York, American Association of Wine Economists.

Rosen, S. (1974), "Hedonic prices and implicit markets: product differentiation in pure competition", Journal of Political Economy, vol. 82, $\mathrm{N}^{\circ}$ 1, Chicago, University of Chicago Press.

Schamel, G. (2002), "California wine winners: a hedonic analysis of regional and winery reputation indicators", Annual Meeting, Long Beach, California, Agricultural and Applied Economics Association, julio.

(2000), "Individual and collective reputation indicators of wine quality", Policy Discussion Paper, № 9, Adelaida, Centre for International Economic Studies, Universidad de Adelaida.

Schamel, G. y K. Anderson (2003), "Wine quality and varietal, regional and winery reputations: hedonic prices for Australia and New Zealand", Economic Record, vol. 79 N ${ }^{\circ} 246$, Wiley Blackwell.

Shapiro, C. (1983), "Premiums for high quality products as returns to reputations", Quarterly Journal of Economics, vol. 98, $\mathrm{N}^{\circ} 4$, Cambridge, Massachusetts, MIT Press.

Stein, S. (2008), "Our saviors may not speak Spanish: changing markets and strategies in Argentina's wine revolution, 1990-2008", AAWE Working Paper, $\mathrm{N}^{\mathrm{o}} 21$, Nueva York, American Association of Wine Economists. 
Tirole, J. (1996), "A theory of collective reputations (with applications to the persistence of corruption and to firm quality", Review of Economic Studies, vol. 63, No 1, Wiley Blackwell.

Van Tienhoven, A. (2008), "The global wine industry: how small Chilean wineries should compete", Amsterdam, Centro Interuniversitario de Estudios y Documentación Latinoamericanos, Universidad de Amsterdam.

Winfree, J. y J. McCluskey (2005), "Collective reputation and quality", American Journal of Agricultural Economics, vol. 87, $\mathrm{N}^{\circ} 1$, Hoboken, Wiley Blackwell. 